\title{
Prediction of Seizure Recurrence. A Note of Caution
}

\author{
William J. Bosl ${ }^{1,2,3 *}$, Alan Leviton ${ }^{1,2}$ and Tobias Loddenkemper ${ }^{1,2}$ \\ ${ }^{1}$ Boston Children's Hospital, Boston, MA, United States, ${ }^{2}$ Harvard Medical School, Boston, MA, United States, ${ }^{3}$ Health \\ Informatics Program, University of San Francisco, San Francisco, CA, United States
}

Great strides have been made recently in documenting that machine-learning programs can predict seizure occurrence in people who have epilepsy. Along with this progress have come claims that appear to us to be a bit premature. We anticipate that many people will benefit from seizure prediction. We also doubt that all will benefit. Although machine learning is a useful tool for aiding discovery, we believe that the greatest progress will come from deeper understanding of seizures, epilepsy, and the EEG features that enable seizure prediction. In this essay, we lay out reasons for optimism and skepticism.

Keywords: electroencephalography, machine learning, chaos \& non-linearity, dynamical systems, seizure prediction

\section{INTRODUCTION}

\section{OPEN ACCESS}

Edited by:

Fernando Cendes,

State University of Campinas, Brazil

Reviewed by:

Kais Gadhoumi,

Duke University, United States Hamid Soltanian-Zadeh,

University of Tehran, Iran

*Correspondence:

William J. Bos

william.bos/@childrens.harvard.edu

Specialty section:

This article was submitted to

Epilepsy,

a section of the journal

Frontiers in Neurology

Received: 03 March 2021 Accepted: 20 April 2021

Published: 13 May 2021

Citation:

Bos/ WJ, Leviton A and Loddenkemper T (2021) Prediction of

Seizure Recurrence. A Note of Caution. Front. Neurol. 12:675728. doi: 10.3389/fneur.2021.675728
In a 2016 Epilepsy Foundation research program survey of people with epilepsy, "unpredictability was selected as a top issue regardless of seizure frequency or severity" (1). Seizure recurrence can be severely limiting (e.g., no driving), (2) socially disruptive and stigmatizing (3), and even life-threatening (4). Consequently, seizure prediction has the potential to improve epilepsy management and, therefore, the quality of life of persons with epilepsy (5).

Successful methods to predict an imminent seizure based on electrographic signatures, and potentially intervene with, for example, "responsive neurostimulation" (RNS) (6-8), would allow alternatives to drugs to minimize seizure recurrence. When a "pro-ictal" $(9,10)$ or "pre-ictal state" $(11,12)$ is identified, this mode of therapy provides highly localized stimulation intended to interrupt a seizure. Identification of times of greater and lesser seizure susceptibility will likely benefit patients, as such states may permit urgent care and interventions.

Progress in our understanding of how seizures develop and propagate (13) would lead to the expectation that "it may be possible to provide seizure prediction to a wider range of patients than previously thought" (14). We are not so sure. Before we explain why we expect only limited success anytime soon, we briefly review what is new with seizure prediction and potential electronic interventions for refractory epilepsy.

Clinicians and patients have long known that some seizures can be preceded by warning signs or symptoms (15). By and large, only about a quarter of patients with generalized epilepsy acknowledge an aura (15).

Electrocorticography (ECoG), which records from electrodes placed directly on the exposed surface of the brain, appears to be the best way to gather all the information for surgical removal of a seizure focus (16). ECoG signals are physically identical to EEG signals. Since they are placed directly on the cortex, less noise contaminates the signal and electrodes can be more closely spaced. Otherwise, the raw data from ECoG that might be fed into a machine learning algorithm is the same as with EEG data. The prediction of seizure recurrence now seems possible with scalp electrodes (17-31). Nevertheless, "modest outcomes associated with localization of abnormal 
electrophysiology suggest ... a fundamental gap in our understanding of how neurophysiologic biomarkers relate to pathophysiology" (32).

Seizure prediction algorithms can be characterized in a variety of ways. One perspective is to consider three independent aspects or axes: (1) the physiological signal to be measured, such as brain electrical activity or heart rate; (2) signal processing methods, which compute various signal features; and (3) machine learning methods, which take signal features as input and attempt to find patterns of features that distinguish seizure activity from non-seizure activity. Advances in signal features and machine learning algorithms continue to advance rapidly and contribute to improved seizure prediction. Signal features that are associated with seizures appear to be patient-specific. Hence, to have any value, machine learning algorithms need signal features from many seizures over a long period of time (33). EEG measures of brain electrical activity continue to be the most common physiological measure associated with seizures, but other measures based on cardiac function, dermal response, or movement are also used (34).

\section{BACKGROUND FOR UNDERSTANDING SEIZURE GENERATION, INHIBITION, AND PROPAGATION}

In the next few paragraphs, we review some elements of seizure generation and propagation that might aid in understanding electrographic correlates of seizures.

\section{Epileptogenic Zone}

The epileptogenic zone (EZ) is tautologically defined as the brain area indispensable for seizure generation (35). Among patients with focal epilepsy, more than $90 \%$ of seizures have discharges in the seizure focus and not elsewhere (36). In some patients, however, complete resection of the presumed EZ did not lead to seizure-freedom (37). Post-surgical recordings of these patients suggest that areas adjacent to the resection were also triggering the epileptic seizures. So was born the concept of "potential seizure-onset zones" (37).

Deeper gray structures (such as the thalamic reticular nucleus) appear to modulate the onset and propagation of other seizure phenomena (e.g., epileptic $2-4 \mathrm{~Hz}$ spike-wave discharges) (38). In addition, the epileptogenic zone in patients with pharmacoresistant seizures can be larger than in people whose seizures are more readily controlled with medication (39).

\section{Seizure Propagation and Networks}

Seizure generation is only the beginning. Seizures are propagated "when synchronous connected groups of neurons work in tandem with rapidly changing de-synchronous relationships from the surrounding epileptic network" (40). The balance between inhibition and propagation, and-to a certain extentunderlying structural and functional connectivity, will determine to what extent the seizure does or does not spread (41). One seizure onset pattern is characterized by hypersynchrony and progressive impairment of inhibition leading to seizure propagation (42).

Seizures are currently defined by the area and signal recorded. As identification of these improve, so will seizure definition and seizure detection. Examples are intensive care patients who had a much higher percentage of seizures detected by intracortical depth electrodes than by surface EEG (43). Therefore, higher spatial resolution, and evaluation of additional signal characteristics have the potential to influence our perception of seizures. Hence, seizure prediction hinges on our definition of seizure onset, which is likely to change as detection techniques improve.

Inter-neuronal activity in the cortex can restrain the spread of epileptiform activity (44). As might be expected, seizure propagation is enhanced when local inhibition networks are defective (45).

Although many reports of brain functional connectivity have assumed "temporal stationarity" (i.e., no change with time), brain networks do reorganize almost continuously in response to both internal and external stimuli, resulting in temporal fluctuations of functional connectivity within and between networks across multiple time-scales $(46,47)$. By "coordinating excitability between brain regions in the epileptic network," changes in functional connectivity between/among networks not only allow propagation of the seizure activity, but might "enhance initiation, evolution, and termination of seizures" (32). The widespread disturbances of structural and functional connectivity that characterize some seizure disorders also appear to contribute to treatment resistance (48).

Epilepsy is considered to be a disorder of neural network organization (49). Research in network science has shown that small changes in network structure can have very large effects on network function, just as small changes in initial conditions can have large effects on time series (50). This suggests that small changes to a non-epileptic neural network may be all that's needed to make the brain epileptic. Similarly, small changes in just the right brain regions may be all that's needed to reduce seizures. Although this has not yet been demonstrated in humans, tools for measuring functional cortical networks are now available (51).

The signal variability of local connectivity among people with epilepsy appears to be significantly higher than in healthy controls (52), bringing excitability of the cortical neurons more often closer to the tipping point of seizures. Although network connectivity in seizure-onset zones can be increased during inter-ictal epochs $(32,53)$, ictal electrographic patterns appear to be generated by network mechanisms that are different from those sustaining inter-ictal potentials (54). Even brief focal spikes can activate diffuse distant networks (55), supporting the characterization of epilepsy as a network disease $(56,57)$.

\section{Electrographic Correlates/Patterns /Signatures of Seizures}

In one third of patients with a diagnosis of pharmaco-resistant focal epilepsy who are candidates for therapeutic surgery, fast activity at $80-120 \mathrm{~Hz}$ associated with very slow transient 
polarizing shift, and voltage depression appear to be excellent biomarkers of ictogenesis and reliable indicators of epileptogenic zone boundaries (58). The high rate of co-occurrence probably reflects the restrictive criteria used to select candidates for surgery who have a presumed single-seizure-focus. Others have found spectral power in discreet frequency bands, as well as time- and/or frequency-domain inter-channel correlations to be helpful $(14,59)$.

Still other seizure onset patterns are characterized by desynchronization of background activity and the appearance of fast low-voltage rhythms (41, 42), while excessive synchronization correlates with termination of the seizure (60). The seizure evolution pathway appears to differ among patients and tends to be stereotypical for each individual $(11,13,61)$. Consequently, for prediction purposes, ictal electrographic signatures need to be individualized for each person for each seizure type $(5,14,17,20,23,25,31,56,62-72)$. The buzzword is "patient-specific." Perhaps "big data" should be another buzz-word because analyses of large sample sizes and multiple individual variables will be needed to decide if groups of patients with similar epilepsy types and other physiological or demographic conditions can be viewed as a (relatively homogeneous) group.

\section{Chaos and Chaotic Systems}

Unlike its meaning in common parlance, "chaos" does not mean random, but only practically unpredictable. Even though the current state of the system might be known almost infinitely precisely, the smallest error or perturbation limits our ability to predict future states of the system.

Seizures often appear to be surprising. This apparent unpredictability might reflect purely random phenomena, or emergent chaotic phenomena that can arise at any time. If seizures are random, then prediction may be impossible in most cases until the pre-seizure changes begin to occur. If seizures are emergent chaotic phenomena, seizure prediction should be possible, since chaotic systems are deterministic. However, the chaotic nature of the system may limit the pre-seizure prediction time.

Non-linear (or chaotic) systems are composed of parts that can interact in complex ways, even if the parts themselves have simple dynamics or behavior. Non-linear systems are characterized by sensitive dependence on initial conditions, emergent phenomena, spontaneous order or synchronization between components, adaptation, and feedback loops (defined below), all of which result from the complex interaction of the parts. The EEG patterns of epilepsy appear to be non-linear $(73,74)$, likely reflecting non-linear dynamics of the brain.

Emergence has been defined as "the arising of novel and coherent structures, patterns and properties during the process of self-organization in complex systems" (75). This process of "self-organization" consists of adaptive behaviors between parts that emerge within chaotic systems, leading to a limited number of relatively stable configurations (76). The non-epileptic brain is stable and does not easily move into an ictal (seizure) state. It exhibits a property called "dynamical resistance" to seizures, which refers to a resistance to transitions to a seizure state (77). Resilience, a similar dynamical property, describes a system's ability to maintain normal function when internal errors or external environmental conditions arise (78). The epileptic brain may have reduced dynamical resistance and/or resilience, resulting in "multistable dynamics," (79) which means that it may spontaneously self-organize into a stable ictal state $(80,81)$. Dynamic networks based on EEG channel synchrony or coherence (amplitude synchrony) of the EEG may also differentiate patients with generalized epilepsy from normal controls (82).

Sensitive dependence on initial conditions is exemplified by the butterfly effect. In the highly non-linear atmospheric system, a small perturbation produced by a butterfly can lead to large changes at a future time, perhaps even a hurricane. In short, an arbitrarily small change in the state of a non-linear system at one time can have a large effect later. This is what makes a deterministic non-linear system practically unpredictable. It is not yet known if seizure occurrence (as opposed to the underlying neural spiking activity) follows a deterministic, chaotic pattern, or if it is simply a purely random process $(83,84)$.

Nobody is in charge of food distribution for most major cities and yet food gets distributed. This characteristic of complex systems is identified as spontaneous order, which may represent what occurs during the inter-ictal resting state (85). Another perspective is that the ictal and inter-ictal states each represent a stable, or semi-stable, attractor state of the dynamical system. An epileptic brain transitions between these states relatively easily, while this phase shift is very difficult to induce in a non-epileptic brain.

Neural connectivity, information transmission, and processing that are essential functions of the brain, may be altered on a large scale to allow the brain to switch into pathological states such as seizures, suggesting a scale dependent tipping (critical) point between normal physiologic function and pathological spread of electrical activity (86). However, if the neural structure of the brain is near a critical point, small changes in neural network structure may tip the brain into an unstable regime where seizures can occur spontaneously. This kind of spatial sensitivity to small changes has been described for networks (87).

\section{Pre-ictal}

If seizure prediction is to become clinically useful, programs that analyze electrical activity need to identify the pre-ictal state as early and reliably as possible before seizure onset. At present, we do not know when the pre-ictal state begins. Knowing when the pre-ictal state begins will allow an assessment of the time needed to detect and interrupt an impending seizure.

Dynamic models of events define different phase transitions (some with and others without an event or characteristic) and then model the probability of transitions from one state to another (88-91). People who work on seizure-prediction algorithms recognize at least three states: a seizure (ictal) state, a pre- or pro-ictal state, and all others. Machine-learning programs are given the task of comparing the electrographic characteristics of variously defined time intervals before a seizure to the electrographic characteristics of times further away (in time) 
from seizure onset. The goal is to define a pre-ictal state. To do this effectively, the machine-learning programs need to be provided an abundance of EEG recordings (92), which are becoming increasingly available.

\section{Characteristics of Ictal EEG}

One group found that a few hours before a seizure, the "network states become less variable ("degenerate"), and this phase is followed by a global functional connectivity reduction" (93). Others have reported "less chaos" $(94,95)$ or "increased synchronization" before a seizure $(18,96)$. One group found that prior to seizure onset, the amplitude of pre-ictal discharges progressively increased as the interval between these discharges gradually decreased (97), while others have found that the cumulative energy profile (98), or measures of spectral entropy, spectral energy, and signal energy can help identify preictal states (17). Still others have emphasized that the best discriminators vary for each individual (99), while another group emphasized the co-occurrence of multiple phenomena in a high potassium hippocampal slice model (loss of neuronal network resilience within the setting of critical slowing down, decreased ability of a network to recover from perturbations, increased high frequency fast activity, and successively decreasing resilience to stimulation (100).

\section{Timing}

The goal is to be able to identify the increased seizure propensity sufficiently before the seizure onset. The interval between identification of the likelihood of an impending seizure and the occurrence of the seizure has varied considerably, from under $10 \mathrm{~s}(17,23,24,72,81,101,102)$ to intervals of an hour or more $(20-22,36,93,103)$.

\section{Periodicity}

Seizures can display multiple types of periodicities (e.g., circadian, multi-day, weekly) in dogs (104) and humans (5, 105109). Because only some people have seizures that occur with an obvious periodicity, seizure prediction is best viewed as patient-specific (5). Changes in level of epileptogenicity (state transitions $)(110,111)$ that most likely characterize periodicities are best viewed as contributing information to seizure-forecasts (112). To what extent these periodicities reflect changes in high-frequency oscillations (112), EEG spike potentials (112), brain connectivity, and inhibitory neurons (113) remains to be quantified. Seizure prediction algorithms are most likely to be effective when they include all the variables that provide relevant discriminating information for that patient. Each individual's seizure periodicities, once quantified, may be among those discriminating information.

\section{Warning Signals Before Critical Transitions}

The existence of early warning signals before catastrophes (e.g., species extinction, pandemics) (114-117) supports the concept that gradual transitions from stable to unstable conditions can reach a tipping point that heralds the irreversibility of the transition (118). Phase transitions in chaotic systems can happen either gradually or suddenly, depending on the system (119).
Indeed, the relatively early aspects of the transition from a nonseizure state to seizure activity can be gradual $(54,120,121)$ and widespread (36). "The suitability of typically applied early warning indicators for identifying heightened probability of a seizure remains controversial" $(122,123)$.

\section{Binary Forecast or Probability Estimate of Seizure Risk}

The seizure detection system can provide a binary forecast (impending seizure: yes/no), or a forecast that provides an estimated probability of an impending seizure $(91,124)$. The probability forecast, though obviously more informative than a binary forecast, will likely be degraded to a binary forecast when algorithms are written to initiate responsive neurostimulation (8). Even binary forecasting systems (high- and low-risk), using only patient-reported seizure data, correctly predicted seizures in about half of 50 patients (125).

\section{Relatively Reliable Prediction}

In an international crowdsourcing competition, an appreciable number of the more than 10,000 algorithms submitted by 478 teams were able "to distinguish between 10-min inter-seizure versus pre-seizure data clips" for each of three patients based on 442 days of continuous intracranial electroencephalography recordings from 16 subdural electrodes (14). These results prompted the authors to conclude, "clinically-relevant seizure prediction is possible in a wider range of patients than previously thought possible." While these results are promising, they are limited to three patients. As noted previously, different patients, or different epilepsy types, may have different pre-ictal time periods, ranging from seconds to an hour or more. Much larger patient sample populations will be needed to map out the limits of pre-seizure prediction. As a first step in this direction, crowdsourcing analysis of intracranial EEGs continues on related platforms, such as epilepsyecosystem.org $(14,126)$.

\section{Not so Reliable Prediction}

Despite subsequent expressions of enthusiasm (12, 31, 92, 127, 128), others have found that the EEGs of one third of patients with focal (129) or multifocal (130) epilepsies were not able to provide adequate predictive information about impending seizures. Findings such as these prompt us to offer words of caution about the anticipated capability to predict seizures and intervene effectively to prevent seizure occurrence.

In our acknowledging that some, perhaps many, people with seizures will benefit from machine-learning programs that predict seizure recurrence, we also want to justify the restraint in our enthusiasm. We do so based on the following considerations.

\section{Prediction Performance Metrics}

Specification of system parameters, such as prediction period, prediction horizon and data-driven characterization of lead seizures (minimal duration of seizure-free period) each influence prediction performance metrics (131). Consequently, investigators have the opportunity to cherrypick the system parameters that will variably maximize their metrics. To minimize this, one group proposed a test 
metric of the difference between algorithm sensitivity and chance sensitivity given an equal proportion of time spent under warning (132).

Prediction performance metrics may include indicators of sensitivity and specificity (59). Sensitivity is defined as the total number of seizures being accurately predicted divided by the total number of seizures recorded. Specificity is the number of correctly-identified non-events and is usually more difficult to evaluate due to the relatively small number of seizure events during most time intervals (133). Performance indices related to specificity include time in warning (the fraction of time the system makes positive predictions), and false positive error rate $(8,132)$.

A more general measure of performance that summarizes the tradeoff between sensitivity and specificity is the area under the receiver operating characteristic (ROC) curve (AUC) that discriminates between inter-ictal and pre-ictal data and is the preferred measure for many studies benchmarking multiple seizure forecasting algorithms $(59,132,134)$.

The ROC curve is a plot of True Positive Rate (TPR) or sensitivity, vs. the False Positive Rate (FPR), or 1-specificity for varying model parameters. Thus, the area under the ROC curve (AUC) is a measure that accounts for the relative tradeoff between sensitivity and specificity. Both are needed for a prediction algorithm to be practical. For example, perfect sensitivity is always possible if specificity is completely sacrificed: always predict a seizure and every seizure will be correctly predicted, $100 \%$ of the time. Similarly, always predicting "no seizure" will never falsely predict a seizure and thus have perfect specificity. Clearly, neither of these extremes is useful. Optimizing both sensitivity (predict all seizures) and specificity (no false alarms) is the ideal. The AUC is a measure of this optimal balance (135).

Because of the potential problem of overfitting (136) of the evaluation statistical model, investigators now seek to measure an "optimism corrected AUC," which corrects for/avoids optimism by either: cross-validation with replication (137-139) or leavepair-out cross-validation (140).

Variations or extensions of this theme include a final "Improvement over Chance" binary metric that compares the measured AUC to a "chance-level AUC" (141), accuracy rates based on ROC curves (142), and an ROC analysis to extrapolate a cut-off value for the most significant predictors of seizure recurrence (143).

Another potential approach to assessing the accuracy of a prediction algorithm is to compare its accuracy to that obtained using surrogate output data that has some of the properties of the true data. An example of this is to randomly permute or shuffle the outcomes labels, thus retaining the same number of positives and negatives as in the original outcomes. (144). After permuting the labels, the predictive accuracy, including sensitivity and specificity, is computed. This process is repeated many times in a Monte Carlo style simulation, and the accuracies for all of the surrogate trials are accumulated to determine how likely the predictive accuracy with the true labels can be attained by random chance. For reasons that are not clear, this type of Monte Carlo simulation, which can be used to estimate the probability of attaining a selected AUC (145) has been used less frequently (146-148).

\section{DISCUSSION}

\section{Butterfly Effect/Important Data Missing}

The butterfly effect refers to the sensitive dependence of a nonlinear system on the accuracy of measurements at a given starting time. Prediction of the future state of a non-linear system is limited by the butterfly effect. For example, even if all of the exact physical equations for atmospheric dynamics are known, predicting the weather more than a few days into the future is limited by how accurately the present weather conditions can be measured at every location from the surface of the earth to the top of the lower atmosphere. If neural function is a nonlinear system, then seizure prediction may be limited by the butterfly effect.

The butterfly effect results from the slightest measurement imprecision. This is different from a lack of information about all the important processes involved in seizure generation or a lack of data. We prefer to use the word "missingness" $(149,150)$ to describe a lack of measured data regarding the processes involved in seizure onset and spread.

Seizure prediction will enable successful intervention only if identifiable pre-ictal signatures occur sufficiently clearly and sufficiently early to enable a predictive model to be constructed. A few reviews of the many applications of signal processing and predictive algorithms present the enormous breadth of this effort (151-154). This approach has begun to be applied to seizure prediction $(155,156)$ with the recognition that the amount of raw EEG data needed for deep learning approaches might be prohibitively large (157).

\section{One Size Does Not Fit All}

An algorithm created for one person is unlikely to predict seizure recurrence in another $(5,11,13,14,17,20,23,25,31$, $56,61-72)$. Another potential problem is that although seizure prediction is specific to an epilepsy or seizure type, prediction can be conditioned by myriad patient characteristics. Large amounts of patient data, together with properly used machine learning algorithms, are likely needed to identify the best way to apply seizure prediction for optimal patient benefit. Sufficient amounts of data from many patients may improve the ability of patient-independent algorithms for the benefit of patients and their physicians who would strongly prefer not to have to wait a year to receive benefits from the prediction capability of wearable devices. However, it is also clear that seizure prediction algorithms can learn from patient-specific patterns and improve over time scales from days to months (158-160).

Much of the success of the seizure prediction field is owed to those investigators who have created a valuable database, made it publicly available, and asked others to contribute to this culture of data sharing (161). Many annotated seizure databases exist. Some of the better known ones can be explored further in these references: $(71,125,161-165)$.

Research using machine learning algorithms is frequently hampered because of the lack of standards that allow data from 
disparate databases to be aggregated (166). A lack of sufficient amounts of publicly-available data is also apparent (154). Because insufficient available human data were (59), a recent Kaggle machine learning competition for seizure prediction relied on canine EEG data. Advances in seizure prediction will be enhanced if the epilepsy research community can collaborate to create a common, aggregated, publicly-available data resource as the genomics community has done for the Human Genome Project (167).

We are cautiously optimistic that many people will benefit from an ability to predict seizure recurrence. We do, however, want to temper optimism that this ability will be available to nearly all patients and all seizures. Very large seizure data sets, with proper clinical annotation, and machine learning algorithms, as well as deeper understanding of the dynamics and neurophysiology of seizures and epilepsy, will be needed to

\section{REFERENCES}

1. Dumanis SB, French JA, Bernard C, Worrell GA, Fureman BE. Seizure forecasting from idea to reality. Outcomes of the My Seizure Gauge Epilepsy Innovation Institute Workshop. eNeuro. (2017) 4:1-5. doi: 10.1523/ENEURO.0349-17.2017

2. Al Zaid EH. Prevalence of patients with epilepsy unfit to drive. J Fam Comm Med. (2019) 26:51-6. doi: 10.4103/jfcm.JFCM_177_17

3. Besag FMC, Vasey MJ. Social cognition and psychopathology in childhood and adolescence. Epilepsy Behav. (2019) 100:106210. doi: 10.1016/j.yebeh.2019.03.015

4. DeGiorgio CM, Curtis A, Hertling D, Moseley BD. Sudden unexpected death in epilepsy: risk factors, biomarkers, and prevention. Acta Neurol Scand. (2019) 139:220-30. doi: 10.1111/ane.13049

5. Amengual-Gual M, Sanchez Fernandez I, Loddenkemper T. Patterns of epileptic seizure occurrence. Brain Res. (2019) 1703:3-12. doi: 10.1016/j.brainres.2018.02.032

6. Matias $\mathrm{CM}$, Sharan $\mathrm{A}, \mathrm{Wu}$ C. Responsive neurostimulation for the treatment of epilepsy. Neurosurg Clin N Am. (2019) 30:231-42. doi: 10.1016/j.nec.2018.12.006

7. Singhal NS, Numis AL, Lee MB, Chang EF, Sullivan JE, Auguste KI, et al. Responsive neurostimulation for treatment of pediatric drug-resistant epilepsy. Epilepsy Behav Case Rep. (2018) 10:21-4. doi: 10.1016/j.ebcr.2018.02.002

8. Gadhoumi K, Lina JM, Mormann F, Gotman J. Seizure prediction for therapeutic devices: a review. J Neurosci Methods. (2016) 260:27082. doi: 10.1016/j.jneumeth.2015.06.010

9. Lopes da Silva FH, Harding GF. Transition to seizure in photosensitive epilepsy. Epilepsy Res. (2011) 97:27882. doi: 10.1016/j.eplepsyres.2011.10.022

10. Baud MO, Proix T, Rao VR, Schindler K. Chance and risk in epilepsy. Curr Opin Neurol. (2020) 33:163-72. doi: 10.1097/WCO.0000000000000798

11. Badawy RA, Freestone DR, Lai A, Cook MJ. Epilepsy: everchanging states of cortical excitability. Neuroscience. (2012) 222:89-99. doi: 10.1016/j.neuroscience.2012.07.015

12. Luckett P, Pavelescu E, McDonald T, Hively L, Ochoa J. Predicting state transitions in brain dynamics through spectral difference of phase-space graphs. J Comp Neurosci. (2019) 46:91-106. doi: 10.1007/s10827-0180700-1

13. Karoly PJ, Kuhlmann L, Soudry D, Grayden DB, Cook MJ, Freestone DR. Seizure pathways: a model-based investigation. PLoS Comp Biol. (2018) 14:e1006403. doi: 10.1371/journal.pcbi.1006403

14. Kuhlmann L, Karoly P, Freestone DR, Brinkmann BH, Temko A, Barachant A, et al. Epilepsyecosystem.org: crowd-sourcing reproducible seizure prediction with long-term human intracranial EEG. Brain. (2018) 141:261930. doi: 10.1093/brain/awy210 provide a much clearer picture of the limits and possibilities of seizure prediction.

\section{DATA AVAILABILITY STATEMENT}

The original contributions presented in the study are included in the article/supplementary material, further inquiries can be directed to the corresponding author/s.

\section{AUTHOR CONTRIBUTIONS}

$\mathrm{AL}$ and TL conceived of the original idea for this paper. AL wrote the first draft. WB wrote substantial sections on non-linear systems, chaos theory, and EEG processing. All authors contributed to the article and approved the submitted version.

15. Perven G, So NK. Epileptic auras: phenomenology and neurophysiology. Epileptic Disord. (2015) 17:349-62. doi: 10.1684/epd.2015.0786

16. King-Stephens D, Mirro E, Weber PB, Laxer KD, Van Ness PC, Salanova V, et al. Lateralization of mesial temporal lobe epilepsy with chronic ambulatory electrocorticography. Epilepsia. (2015) 56:959-67. doi: 10.1111/epi.13010

17. Sridevi V, Ramasubba Reddy M, Srinivasan K, Radhakrishnan K, Rathore C, Nayak DS. Improved patient-independent system for detection of electrical onset of seizures. J Clin Neurophysiol. (2019) 36:1424. doi: 10.1097/WNP.0000000000000533

18. Li F, Liang Y, Zhang L, Yi C, Liao Y, Jiang Y, et al. Transition of brain networks from an interictal to a preictal state preceding a seizure revealed by scalp EEG network analysis. Cogn Neurodyn. (2019) 13:17581. doi: 10.1007/s11571-018-09517-6

19. Khoa TQ, Huong NT, Toi VV. Detecting epileptic seizure from scalp EEG using Lyapunov spectrum. Comp Math Methods Med. (2012) 2012:847686. doi: 10.1155/2012/847686

20. Minasyan GR, Chatten JB, Chatten MJ, Harner RN. Patient-specific early seizure detection from scalp electroencephalogram. J Clin Neurophysiol. (2010) 27:163-78. doi: 10.1097/WNP.0b013e3181e0a9b6

21. Yang Y, Zhou M, Niu Y, Li C, Cao R, Wang B, et al. Epileptic seizure prediction based on permutation entropy. Front Comp Neurosci. (2018) 12:55. doi: $10.3389 /$ fncom.2018.00055

22. Saab ME, Gotman J. A system to detect the onset of epileptic seizures in scalp EEG. Clin Neurophysiol. (2005) 116:42742. doi: 10.1016/j.clinph.2004.08.004

23. Zandi AS, Javidan M, Dumont GA, Tafreshi R. Automated real-time epileptic seizure detection in scalp EEG recordings using an algorithm based on wavelet packet transform. IEEE Transact Biomed Eng. (2010) 57:163951. doi: 10.1109/TBME.2010.2046417

24. Salant Y, Gath I, Henriksen O. Prediction of epileptic seizures from two-channel EEG. Med Biol Eng Comp. (1998) 36:54956. doi: 10.1007/BF02524422

25. Alotaiby TN, Alshebeili SA, Alotaibi FM, Alrshoud SR. Epileptic seizure prediction using CSP and LDA for scalp EEG signals. Comp Intell Neurosci. (2017) 2017:1240323. doi: 10.1155/2017/1240323

26. Zibrandtsen IC, Kidmose P, Christensen CB, Kjaer TW. Ear-EEG detects ictal and interictal abnormalities in focal and generalized epilepsy A comparison with scalp EEG monitoring. Clin Neurophysiol. (2017) 128:2454-61. doi: 10.1016/j.clinph.2017.09.115

27. Jin K, Nakasato N. Long-cherished dreams for epileptologists and clinical neurophysiologists: automatic seizure detection in long-term scalp EEG. Clin Neurophysiol. (2014) 125:1289-90. doi: 10.1016/j.clinph.2013. 12.105

28. Hopfengartner R, Kasper BS, Graf W, Gollwitzer S, Kreiselmeyer G, Stefan $\mathrm{H}$, et al. Automatic seizure detection in long-term scalp EEG using an adaptive thresholding technique: a validation study for clinical 
routine. Clin Neurophysiol. (2014) 125:1346-52. doi: 10.1016/j.clinph.2013. 12.104

29. Nemtsas P, Birot G, Pittau F, Michel CM, Schaller K, Vulliemoz S, et al. Source localization of ictal epileptic activity based on high-density scalp EEG data. Epilepsia. (2017) 58:1027-36. doi: 10.1111/epi.13749

30. Lam AD, Maus D, Zafar SF, Cole AJ, Cash SS. SCOPE-mTL: a noninvasive tool for identifying and lateralizing mesial temporal lobe seizures prior to scalp EEG ictal onset. Clin Neurophysiol. (2017) 128:164755. doi: 10.1016/j.clinph.2017.06.040

31. Detti P, de Lara GZM, Bruni R, Pranzo M, Sarnari F, Vatti G. A patientspecific approach for short-term epileptic seizures prediction through the analysis of EEG synchronization. IEEE Transact Biomed Eng. (2019) 66:1494-504. doi: 10.1109/TBME.2018.2874716

32. Khambhati AN, Bassett DS, Oommen BS, Chen SH, Lucas TH, Davis $\mathrm{KA}$, et al. Recurring functional interactions predict network architecture of interictal and ictal states in neocortical epilepsy. eNeuro. (2017) 4:118. doi: 10.1523/ENEURO.0091-16.2017

33. Manzouri F, Heller S, Dumpelmann M, Woias P, Schulze-Bonhage A. A comparison of machine learning classifiers for energy-efficient implementation of seizure detection. Front Syst Neurosci. (2018) 12:43. doi: $10.3389 /$ fnsys.2018.00043

34. Tsiouris KM, Tzallas AT, Markoula S, Koutsouris D, Konitsiotis S, Fotiadis DI. A review of automated methodologies for the detection of epileptic episodes using long-term eeg signals. Healthcare Pol Reform Concepts Methodol Tools Appl. (3201) 8:1464-96. doi: 10.4018/978-1-5225-6915-2.ch066

35. Rosenow F, Bast T, Czech T, Feucht M, Hans VH, Helmstaedter C, et al. Revised version of quality guidelines for presurgical epilepsy evaluation and surgical epilepsy therapy issued by the Austrian, German, and Swiss working group on presurgical epilepsy diagnosis and operative epilepsy treatment. Epilepsia. (2016) 57:1215-20. doi: 10.1111/epi.13449

36. Naftulin JS, Ahmed OJ, Piantoni G, Eichenlaub JB, Martinet LE, Kramer $\mathrm{MA}$, et al. Ictal and preictal power changes outside of the seizure focus correlate with seizure generalization. Epilepsia. (2018) 59:1398409. doi: 10.1111/epi.14449

37. Luders HO, Najm I, Nair D, Widdess-Walsh P, Bingman W. The epileptogenic zone: general principles. Epileptic Disord. (2006) 8(Suppl. 2):S1-9.

38. Fan D, Liao F, Wang Q. The pacemaker role of thalamic reticular nucleus in controlling spike-wave discharges and spindles. Chaos. (2017) 27:073103. doi: 10.1063/1.4991869

39. Suzuki $H$, Sugano $H$, Nakajima $M$, Higo $T$, Iimura $Y$, Mitsuhashi $\mathrm{T}$, et al. The epileptogenic zone in pharmaco-resistant temporal lobe epilepsy with amygdala enlargement. Epileptic Disord. (2019) 21:252-64. doi: 10.1684/epd.2019.1075

40. Khambhati AN, Davis KA, Oommen BS, Chen SH, Lucas TH, Litt $\mathrm{B}$, et al. Dynamic network drivers of seizure generation, propagation and termination in human neocortical epilepsy. PLoS Comp Biol. (2015) 11:e1004608. doi: 10.1371/journal.pcbi.1004608

41. Jiruska P, de Curtis M, Jefferys JG, Schevon CA, Schiff SJ, Schindler K. Synchronization and desynchronization in epilepsy: controversies and hypotheses. J Physiol. (2013) 591:787-97. doi: 10.1113/jphysiol.2012. 239590

42. Avoli M, de Curtis M, Gnatkovsky V, Gotman J, Kohling R, Levesque M, et al. Specific imbalance of excitatory/inhibitory signaling establishes seizure onset pattern in temporal lobe epilepsy. J Neurophysiol. (2016) 115:322937. doi: $10.1152 /$ jn. 01128.2015

43. Claassen J, Perotte A, Albers D, Kleinberg S, Schmidt JM, Tu $B$, et al. Nonconvulsive seizures after subarachnoid hemorrhage: multimodal detection and outcomes. Ann Neurol. (2013) 74:53-64. doi: 10.1002/ana.23859

44. Parrish RR, Codadu NK, Mackenzie-Gray Scott C, Trevelyan AJ. Feedforward inhibition ahead of ictal wavefronts is provided by both parvalbumin- and somatostatin-expressing interneurons. J Physiol. (2019) 597:2297-314. doi: 10.1113/JP277749

45. Liou JY, Ma H, Wenzel M, Zhao M, Baird-Daniel E, Smith EH, et al. Role of inhibitory control in modulating focal seizure spread. Brain. (2018) 141:2083-97. doi: 10.1093/brain/awy116
46. Preti MG, Bolton TA, Van De Ville D. The dynamic functional connectome: state-of-the-art and perspectives. Neuroimage. (2017) 160:4154. doi: 10.1016/j.neuroimage.2016.12.061

47. Iraji A, Deramus TP, Lewis N, Yaesoubi M, Stephen JM, Erhardt E, et al. The spatial chronnectome reveals a dynamic interplay between functional segregation and integration. Hum Brain Mapp. (2019) 40:305877. doi: $10.1002 / \mathrm{hbm} .24580$

48. Tavakol S, Royer J, Lowe AJ, Bonilha L, Tracy JI, Jackson GD, et al. Neuroimaging and connectomics of drug-resistant epilepsy at multiple scales: from focal lesions to macroscale networks. Epilepsia. (2019) 60:593604. doi: $10.1111 /$ epi.14688

49. Kramer MA, Cash SS. Epilepsy as a disorder of cortical network organization. Neuroscientist. (2012) 18:360-72. doi: 10.1177/1073858411422754

50. Cornelius SP, Kath WL, Motter AE. Realistic control of network dynamics. Nat Commun. (2013) 4:1942. doi: 10.1038/ncomms2939

51. Carr SJA, Gershon A, Shafiabadi N, Lhatoo SD, Tatsuoka C, Sahoo SS. An integrative approach to study structural and functional network connectivity in epilepsy using imaging and signal data. Front Integr Neurosci. (2020) 14:491403. doi: 10.3389/fnint.2020.491403

52. Pedersen M, Omidvarnia A, Curwood EK, Walz JM, Rayner G, Jackson GD. The dynamics of functional connectivity in neocortical focal epilepsy. NeuroImage Clin. (2017) 15:209-14. doi: 10.1016/j.nicl.2017.04.005

53. Korzeniewska A, Cervenka MC, Jouny CC, Perilla JR, Harezlak J, Bergey GK, et al. Ictal propagation of high frequency activity is recapitulated in interictal recordings: effective connectivity of epileptogenic networks recorded with intracranial EEG. Neuroimage. (2014) 101:96113. doi: $10.1016 /$ j.neuroimage.2014.06.078

54. Huberfeld G, Menendez de la Prida L, Pallud J, Cohen I, Le Van Quyen M, Adam C, et al. Glutamatergic pre-ictal discharges emerge at the transition to seizure in human epilepsy. Nat Neurosci. (2011) 14:62734. doi: $10.1038 / \mathrm{nn} .2790$

55. Walz JM, Pedersen M, Omidvarnia A, Semmelroch M, Jackson GD. Spatiotemporal mapping of epileptic spikes using simultaneous EEGfunctional MRI. Brain. (2017) 140:998-1010. doi: 10.1093/brain/awx007

56. Olmi S, Petkoski S, Guye M, Bartolomei F, Jirsa V. Controlling seizure propagation in large-scale brain networks. PLoS Comp Biol. (2019) 15:e1006805. doi: 10.1371/journal.pcbi.1006805

57. Laufs H. Functional imaging of seizures and epilepsy: evolution from zones to networks. Curr Opin Neurol. (2012) 25:194200. doi: 10.1097/WCO.0b013e3283515db9

58. Gnatkovsky V, de Curtis M, Pastori C, Cardinale F, Lo Russo G, Mai R, et al. Biomarkers of epileptogenic zone defined by quantified stereo-EEG analysis. Epilepsia. (2014) 55:296-305. doi: 10.1111/epi.12507

59. Brinkmann BH, Wagenaar J, Abbot D, Adkins P, Bosshard SC, Chen M, et al. Crowdsourcing reproducible seizure forecasting in human and canine epilepsy. Brain. (2016) 139 (Pt 6):1713-22. doi: 10.1093/brain/aww045

60. Fisher RS, Scharfman HE, deCurtis M. How can we identify ictal and interictal abnormal activity? Adv Exp Med Biol. (2014) 813:323. doi: 10.1007/978-94-017-8914-1_1

61. Jirsa VK, Stacey WC, Quilichini PP, Ivanov AI, Bernard C. On the nature of seizure dynamics. Brain. (2014) $137 \quad(\mathrm{Pt}$ 8):2210-30. doi: 10.1093/brain/awu133

62. Amengual-Gual M, Ulate-Campos A, Loddenkemper T. Status epilepticus prevention, ambulatory monitoring, early seizure detection and prediction in at-risk patients. Seizure. (2019) 68:31-7. doi: 10.1016/j.seizure.2018. 09.013

63. Zabihi M, Kiranyaz S, Jantti V, Lipping T, Gabbouj M. Patient-specific seizure detection using nonlinear dynamics and nullclines. IEEE J Biomed Health Inform. (2020) 24:543-55. doi: 10.1109/JBHI.2019.2906400

64. Selvakumari RS, Mahalakshmi M, Prashalee P. Patient-Specific seizure detection method using hybrid classifier with optimized electrodes. J Med Syst. (2019) 43:121. doi: 10.1007/s10916-019-1234-4

65. Kuhlmann L, Freestone D, Lai A, Burkitt AN, Fuller K, Grayden $\mathrm{DB}$, et al. Patient-specific bivariate-synchrony-based seizure prediction for short prediction horizons. Epilepsy Res. (2010) 91:214-31. doi: 10.1016/j.eplepsyres.2010.07.014

66. Kaleem M, Gurve D, Guergachi A, Krishnan S. Patient-specific seizure detection in long-term EEG using signal-derived empirical mode 
decomposition (EMD)-based dictionary approach. J Neural Eng. (2018) 15:056004. doi: 10.1088/1741-2552/aaceb1

67. Billeci L, Marino D, Insana L, Vatti G, Varanini M. Patient-specific seizure prediction based on heart rate variability and recurrence quantification analysis. PLoS ONE. (2018) 13:e0204339. doi: 10.1371/journal.pone.0204339

68. Sandler RA, Geng K, Song D, Hampson RE, Witcher MR, Deadwyler SA, et al. Designing patient-specific optimal neurostimulation patterns for seizure suppression. Neural Comp. (2018) 30:1180-208. doi: 10.1162/neco_a_01075

69. Bhattacharyya A, Pachori RB. A multivariate approach for patient-specific EEG seizure detection using empirical wavelet transform. IEEE Transact Biomed Eng. (2017) 64:2003-15. doi: 10.1109/TBME.2017.2650259

70. Qu H, Gotman J. A patient-specific algorithm for the detection of seizure onset in long-term EEG monitoring: possible use as a warning device. IEEE Transact Biomed Eng. (1997) 44:115-22. doi: 10.1109/10.552241

71. Shoeb A, Edwards H, Connolly J, Bourgeois B, Treves ST, Guttag J. Patient-specific seizure onset detection. Epilepsy Behav. (2004) 5:48398. doi: 10.1016/j.yebeh.2004.05.005

72. Kharbouch A, Shoeb A, Guttag J, Cash SS. An algorithm for seizure onset detection using intracranial EEG. Epilepsy Behav. (2011) 22(Suppl. 1):S2935. doi: 10.1016/j.yebeh.2011.08.031

73. Lehnertz K. Epilepsy and nonlinear dynamics. J Biol Phys. (2008) 34:25366. doi: 10.1007/s10867-008-9090-3

74. Good LB, Sabesan S, Marsh ST, Tsakalis K, Treiman DM, Iasemidis LD. Nonlinear dynamics of seizure prediction in a rodent model of epilepsy. Nonlinear Dyn Psychol Life Sci. (2010) 14:411-34.

75. Goldstein JM. Emergence as a construct: history and issues. Emergence. (1999) 1:49-72. doi: 10.1207/s15327000em0101_4

76. Rubinov M, Sporns O, Thivierge JP, Breakspear M. Neurobiologically realistic determinants of self-organized criticality in networks of spiking neurons. PLoS Comp Biol. (2011) 7:e1002038. doi: 10.1371/journal.pcbi.1002038

77. Rings T, Mazarei M, Akhshi A, Geier C, Tabar MRR, Lehnertz K. Traceability and dynamical resistance of precursor of extreme events. Sci Rep. (2019) 9:1744. doi: 10.1038/s41598-018-38372-y

78. Gao J, Barzel B, Barabasi AL. Universal resilience patterns in complex networks. Nature. (2016) 530:307-12. doi: 10.1038/nature16948

79. Lopes da Silva FH, Blanes W, Kalitzin SN, Parra J, Suffczynski P, Velis DN. Dynamical diseases of brain systems: different routes to epileptic seizures. IEEE Transact Biomed Eng. (2003) 50:540-8. doi: 10.1109/TBME.2003.810703

80. Freeman WJ, Skarda CA. Spatial EEG patterns, non-linear dynamics and perception: the neo-Sherringtonian view. Brain Res. (1985) 357:14775. doi: 10.1016/0165-0173(85)90022-0

81. Frolov NS, Grubov VV, Maksimenko VA, Luttjohann A, Makarov VV, Pavlov AN, et al. Statistical properties and predictability of extreme epileptic events. Sci Rep. (2019) 9:7243. doi: 10.1038/s41598-019-43619-3

82. Schmidt H, Woldman W, Goodfellow M, Chowdhury FA, Koutroumanidis $\mathrm{M}$, Jewell S, et al. A computational biomarker of idiopathic generalized epilepsy from resting state EEG. Epilepsia. (2016) 57:e200-4. doi: 10.1111/epi.13481

83. Gao J, Hu J, Tung WW, Blasch E. Multiscale analysis of biological data by scale-dependent lyapunov exponent. Front Physiol. (2011) 2:110. doi: $10.3389 /$ fphys.2011.00110

84. Lai YC, Harrison MA, Frei MG, Osorio I. Controlled test for predictive power of Lyapunov exponents: their inability to predict epileptic seizures. Chaos. (2004) 14:630-42. doi: 10.1063/1.1777831

85. Yang T, Fang Z, Ren J, Xiao F, Li Q, Liu L, et al. Altered spontaneous activity in treatment-naive childhood absence epilepsy revealed by regional homogeneity. J Neurol Sci. (2014) 340:58-62. doi: 10.1016/j.jns.2014.02.025

86. Milton JG. Neuronal avalanches, epileptic quakes and other transient forms of neurodynamics. Eur J Neurosci. (2012) 36:2156-63. doi: 10.1111/j.1460-9568.2012.08102.x

87. Motter AE, Albert R. Networks in motion. Phys Today. (2012) 65:438. doi: 10.1063/PT.3.1518

88. McEwen JA, Anderson GB. Modeling the stationarity and Gaussianity of spontaneous electroencephalographic activity. IEEE Transact Biomed Eng. (1975) 22:361-9. doi: 10.1109/TBME.1975.324504
89. Chiang S, Vannucci M, Goldenholz DM, Moss R, Stern JM. Epilepsy as a dynamic disease: a Bayesian model for differentiating seizure risk from natural variability. Epilepsia Open. (2018) 3:236-46. doi: 10.1002/epi4.12112

90. Lopes da Silva F, Blanes W, Kalitzin SN, Parra J, Suffczynski P, Velis DN. Epilepsies as dynamical diseases of brain systems: basic models of the transition between normal and epileptic activity. Epilepsia. (2003) 44(Suppl. 12):72-83. doi: 10.1111/j.0013-9580.2003.12005.x

91. Principe A, Ley M, Conesa G, Rocamora R. Prediction error connectivity: a new method for EEG state analysis. Neuroimage. (2019) 188:26173. doi: 10.1016/j.neuroimage.2018.11.052

92. Acharya UR, Hagiwara Y, Adeli H. Automated seizure prediction. Epilepsy Behav. (2018) 88:251-61. doi: 10.1016/j.yebeh.2018.09.030

93. Tauste Campo A, Principe A, Ley M, Rocamora R, Deco G. Degenerate timedependent network dynamics anticipate seizures in human epileptic brain. PLoS Biol. (2018) 16:e2002580. doi: 10.1371/journal.pbio.2002580

94. Bou Assi E, Nguyen DK, Rihana S, Sawan M. Towards accurate prediction of epileptic seizures: a review. Biomed Signal Process. (2017) 34:14457. doi: 10.1016/j.bspc.2017.02.001

95. Schwartz TH, Hong SB, Bagshaw AP, Chauvel P, Benar CG. Preictal changes in cerebral haemodynamics: review of findings and insights from intracerebral EEG. Epilepsy Res. (2011) 97:252-66. doi: 10.1016/j.eplepsyres.2011.07.013

96. Le Van Quyen M, Soss J, Navarro V, Robertson R, Chavez M, Baulac $\mathrm{M}$, et al. Preictal state identification by synchronization changes in longterm intracranial EEG recordings. Clin Neurophysiol. (2005) 116:55968. doi: 10.1016/j.clinph.2004.10.014

97. Chen J, Li L, Wu D, Li X, Xue Q, Wang L, et al. Dynamic preictal discharges in patients with mesial temporal lobe epilepsy. J Clin Neurophysiol. (2018) 35:381-7. doi: 10.1097/WNP.0000000000000486

98. Litt B, Esteller R, Echauz J, D'Alessandro M, Shor R, Henry T, et al. Epileptic seizures may begin hours in advance of clinical onset: a report of five patients. Neuron. (2001) 30:51-64. doi: 10.1016/S0896-6273(01)00262-8

99. Lin LC, Chen SC, Chiang CT, Wu HC, Yang RC, Ouyang CS. Classification preictal and interictal stages via integrating interchannel and timedomain analysis of EEG features. Clin EEG Neurosci. (2017) 48:13945. doi: $10.1177 / 1550059416649076$

100. Chang WC, Kudlacek J, Hlinka J, Chvojka J, Hadrava M, Kumpost V, et al. Loss of neuronal network resilience precedes seizures and determines the ictogenic nature of interictal synaptic perturbations. Nat Neurosci. (2018) 21:1742-52. doi: 10.1038/s41593-018-0278-y

101. Meier R, Dittrich H, Schulze-Bonhage A, Aertsen A. Detecting epileptic seizures in long-term human EEG: a new approach to automatic online and real-time detection and classification of polymorphic seizure patterns. J Clin Neurophysiol. (2008) 25:119-31. doi: 10.1097/WNP.0b013e3181775993

102. Rogowski Z, Gath I, Bental E. On the prediction of epileptic seizures. Biol Cybernet. (1981) 42:9-15. doi: 10.1007/BF00335153

103. Adames NR, Oberle JR, Cooper JA. The surveillance mechanism of the spindle position checkpoint in yeast. J Cell Biol. (2001) 153:15968. doi: $10.1083 /$ jcb.153.1.159

104. Gregg NM, Nasseri M, Kremen V, Patterson EE, Sturges BK, Denison TJ, et al. Circadian and multiday seizure periodicities, and seizure clusters in canine epilepsy. Brain Commun. (2020) 2:fcaa008. doi: 10.1093/braincomms/fcaa008

105. Baud MO, Kleen JK, Mirro EA, Andrechak JC, King-Stephens D, Chang EF, et al. Multi-day rhythms modulate seizure risk in epilepsy. Nat Commun. (2018) 9:88. doi: 10.1038/s41467-017-02577-y

106. Christodoulakis M, Hadjipapas A, Papathanasiou ES, Anastasiadou M, Papacostas SS, Mitsis GD. Periodicity in functional brain networks: application to scalp EEG from epilepsy patients. Annu Int Conf IEEE Eng Med Biol Sci. (2014) 2014:2805-8. doi: 10.1109/EMBC.2014. 6944206

107. Loddenkemper T, Vendrame M, Zarowski M, Gregas M, Alexopoulos AV, Wyllie E, et al. Circadian patterns of pediatric seizures. Neurology. (2011) 76:145-53. doi: 10.1212/WNL.0b013e318206ca46

108. Hofstra WA, de Weerd AW. The circadian rhythm and its interaction with human epilepsy: a review of literature. Sleep Med Rev. (2009) 13:41320. doi: 10.1016/j.smrv.2009.01.002 
109. Karoly PJ, Goldenholz DM, Freestone DR, Moss RE, Grayden DB, Theodore WH, et al. Circadian and circaseptan rhythms in human epilepsy: a retrospective cohort study. Lancet Neurol. (2018) 17:97785. doi: 10.1016/S1474-4422(18)30274-6

110. Abrishami Shokooh L, Toffa DH, Pouliot P, Lesage F, Nguyen DK. Identification of global and local states during seizures using quantitative functional connectivity and recurrence plot analysis. Comp Biol Med. (2020) 122:103858. doi: 10.1016/j.compbiomed.2020.103858

111. Crisp DN, Cheung W, Gliske SV, Lai A, Freestone DR, Grayden $\mathrm{DB}$, et al. Quantifying epileptogenesis in rats with spontaneous and responsive brain state dynamics. Brain Commun. (2020) 2:fcaa048. doi: 10.1093/braincomms/fcaa048

112. Stirling RE, Cook MJ, Grayden DB, Karoly PJ. Seizure forecasting and cyclic control of seizures. Epilepsia. (2021) 62 Suppl 1:S2-S14. doi: 10.1111/epi.16541

113. Scott JM, Ren S, Gliske SV, Stacey WC. Preictal variability of highfrequency oscillation rates in refractory epilepsy. Epilepsia. (2020) 61:252133. doi: 10.1111/epi.16680

114. Jarvis L, McCann K, Tunney T, Gellner G, Fryxell JM. Early warning signals detect critical impacts of experimental warming. Ecol Evol. (2016) 6:6097-106. doi: 10.1002/ece3.2339

115. Drake JM, Griffen BD. Early warning signals of extinction in deteriorating environments. Nature. (2010) 467:456-9. doi: 10.1038/nature09389

116. Gao J, Wang K, Ding T. Detecting early-warning signals for influenza A pandemic based on protein dynamical network biomarkers. Saudi J Biol Sci. (2017) 24:724-8. doi: 10.1016/j.sjbs.2017.01.048

117. Chen P, Chen E, Chen L, Zhou XJ, Liu R. Detecting early-warning signals of influenza outbreak based on dynamic network marker. J Cell Mol Med. (2019) 23:395-404. doi: 10.1111/jcmm.13943

118. Boettiger C, Hastings A. Tipping points: from patterns to predictions. Nature. (2013) 493:157-8. doi: 10.1038/493157a

119. Solé R. Phase Transitions. Princeton, NJ: Princeton University Press (2011).

120. Zhang ZJ, Koifman J, Shin DS, Ye H, Florez CM, Zhang L, et al. Transition to seizure: ictal discharge is preceded by exhausted presynaptic GABA release in the hippocampal CA3 region. J Neurosci. (2012) 32:2499512. doi: 10.1523/JNEUROSCI.4247-11.2012

121. Worrell GA, Parish L, Cranstoun SD, Jonas R, Baltuch G, Litt B. Highfrequency oscillations and seizure generation in neocortical epilepsy. Brain. (2004) 127 (Pt 7):1496-506. doi: 10.1093/brain/awh149

122. Wilkat $\mathrm{T}$, Rings $\mathrm{T}$, Lehnertz $\mathrm{K}$. No evidence for critical slowing down prior to human epileptic seizures. Chaos. (2019) 29:091104. doi: 10.1063/1. 5122759

123. Maturana MI, Meisel C, Dell K, Karoly PJ, D’Souza W, Grayden DB, et al. Critical slowing down as a biomarker for seizure susceptibility. Nat Commun. (2020) 11:2172. doi: 10.1038/s41467-020-15908-3

124. Mader M, Mader W, Gluckman BJ, Timmer J, Schelter B. Statistical evaluation of forecasts. Phys Rev E Stat Nonlinear Soft Matter Phys. (2014) 90:022133. doi: 10.1103/PhysRevE.90.022133

125. Karoly PJ, Cook MJ, Maturana M, Nurse ES, Payne D, Brinkmann BH, et al. Forecasting cycles of seizure likelihood. Epilepsia. (2020) 61:77686. doi: 10.1111/epi.16485

126. Reuben C, Karoly P, Freestone DR, Temko A, Barachant A, Li F, et al. Ensembling crowdsourced seizure prediction algorithms using longterm human intracranial EEG. Epilepsia. (2020) 61:e7-12. doi: 10.1111/epi. 16418

127. Karuppiah Ramachandran VR, Alblas HJ, Le DV, Meratnia N. Towards an online seizure advisory system-an adaptive seizure prediction framework using active learning heuristics. Sensors. (2018) 18:1-30. doi: 10.3390/s18061698

128. Kuhlmann L, Lehnertz K, Richardson MP, Schelter B, Zaveri HP. Seizure prediction - ready for a new era. Nat Rev Neurol. (2018) 14:61830. doi: 10.1038/s41582-018-0055-2

129. Alexandre Teixeira C, Direito B, Bandarabadi M, Le Van Quyen M, Valderrama M, Schelter B, et al. Epileptic seizure predictors based on computational intelligence techniques: a comparative study with 278 patients. Comp Methods Progr Biomed. (2014) 114:324-36. doi: 10.1016/j.cmpb.2014.02.007
130. Lehnertz K, Dickten H, Porz S, Helmstaedter C, Elger CE. Predictability of uncontrollable multifocal seizures - towards new treatment options. Sci Rep. (2016) 6:24584. doi: 10.1038/srep24584

131. Chen HH, Cherkassky V. Performance metrics for online seizure prediction. Neural Netw. (2020) 128:22-32. doi: 10.1016/j.neunet.2020.04.022

132. Snyder DE, Echauz J, Grimes DB, Litt B. The statistics of a practical seizure warning system. J Neural Eng. (2008) 5:392-401. doi: 10.1088/1741-2560/5/4/004

133. Chaovalitwongse W, Iasemidis LD, Pardalos PM, Carney PR, Shiau DS, Sackellares JC. Performance of a seizure warning algorithm based on the dynamics of intracranial EEG. Epilepsy Res. (2005) 64:93113. doi: 10.1016/j.eplepsyres.2005.03.009

134. Baldassano SN, Brinkmann BH, Ung H, Blevins T, Conrad EC, Leyde $\mathrm{K}$, et al. Crowdsourcing seizure detection: algorithm development and validation on human implanted device recordings. Brain. (2017) 140:168091. doi: 10.1093/brain/awx098

135. Carter JV, Pan J, Rai SN, Galandiuk S. ROC-ing along: evaluation and interpretation of receiver operating characteristic curves. Surgery. (2016) 159:1638-45. doi: 10.1016/j.surg.2015.12.029

136. Lemm S, Blankertz $B$, Dickhaus $T$, Müller KR. Introduction to machine learning for brain imaging. Neuroimage. (2011) 56:387-99. doi: 10.1016/j.neuroimage.2010.11.004

137. La Rocca M, Garner R, Amoroso N, Lutkenhoff ES, Monti MM, Vespa $\mathrm{P}$, et al. Multiplex networks to characterize seizure development in traumatic brain injury patients. Front Neurosci. (2020) 14:591662. doi: 10.3389/fnins.2020.591662

138. Scott JM, Gliske SV, Kuhlmann L, Stacey WC. Viability of preictal highfrequency oscillation rates as a biomarker for seizure prediction. Front Hum Neurosci. (2020) 14:612899. doi: 10.3389/fnhum.2020.612899

139. Weil AG, Lewis EC, Ibrahim GM, Kola O, Tseng C-H, Zhou X, et al. Hemispherectomy outcome prediction scale: development and validation of a seizure freedom prediction tool. Epilepsia. (2021). doi: 10.1111/epi.16861

140. Sip V, Hashemi M, Vattikonda AN, Woodman MM, Wang H, Scholly J, et al. Data-driven method to infer the seizure propagation patterns in an epileptic brain from intracranial electroencephalography. PLoS Comput Biol. (2021) 17:e1008689. doi: 10.1371/journal.pcbi.1008689

141. Proix T, Truccolo W, Leguia MG, Tcheng TK, King-Stephens D, Rao VR, et al. Forecasting seizure risk in adults with focal epilepsy: a development and validation study. Lancet Neurol. (2021) 20:12735. doi: 10.1016/S1474-4422(20)30396-3

142. Kleen JK, Speidel BA, Baud MO, Rao VR, Ammanuel SG, Hamilton LS, et al. Accuracy of omni-planar and surface casting of epileptiform activity for intracranial seizure localization. Epilepsia. (2021) 62:94759. doi: 10.1111/epi.16841

143. Turco F, Bonanni E, Milano C, Pizzanelli C, Steinwurzel C, Morganti R, et al. Prolonged epileptic discharges predict seizure recurrence in JME: insights from prolonged ambulatory EEG. Epilepsia. (2021). doi: 10.1111/epi.16875

144. Golland P, Fischl B. Permutation tests for classification: towards statistical significance in image-based studies. Inf Process Med Imaging. (2003) 18:33041. doi: 10.1007/978-3-540-45087-0_28

145. Ambrose PG, Grasela DM. The use of Monte Carlo simulation to examine pharmacodynamic variance of drugs: fluoroquinolone pharmacodynamics against Streptococcus pneumoniae. Diagn Microbiol Infect Dis. (2000) 38:1517. doi: 10.1016/S0732-8893(00)00185-1

146. Feldwisch-Drentrup H, Schulze-Bonhage A, Timmer J, Schelter B. Statistical validation of event predictors: a comparative study based on the field of seizure prediction. Phys Rev E Stat Nonlin Soft Matter Phys. (2011) 83 (6 Pt 2):066704. doi: 10.1103/PhysRevE.83.066704

147. Struck AF, Rodriguez-Ruiz AA, Osman G, Gilmore EJ, Haider HA, Dhakar MB, et al. Comparison of machine learning models for seizure prediction in hospitalized patients. Ann Clin Transl Neurol. (2019) 6:123947. doi: 10.1002/acn3.50817

148. Andrzejak RG, Chicharro D, Elger CE, Mormann F. Seizure prediction: any better than chance? Clin Neurophysiol. (2009) 120:1465-78. doi: 10.1016/j.clinph.2009.05.019

149. Salgado CM, Azevedo C, Proenca H, Vieira SM. Missing Data. Secondary Analysis of Electronic Health Records. Cham: Springer (2016). p. 143-62. 
150. Sackett DL. Bias in analytic research. J Chronic Dis. (1979) 32:5163. doi: 10.1016/0021-9681(79)90012-2

151. Yang J, Sawan M. From seizure detection to smart and fully embedded seizure prediction engine: a review. IEEE Trans Biomed Circuits Syst. (2020) 14:1008-23. doi: 10.1109/TBCAS.2020.3018465

152. An S, Kang C, Lee HW. Artificial intelligence and computational approaches for epilepsy. J Epilepsy Res. (2020) 10:8-17. doi: 10.14581/jer.20003

153. Boubchir L. Editorial commentary on special issue of advances in EEG signal processing and machine learning for epileptic seizure detection and prediction. J Biomed Res. (2020) 34:149-50. doi: 10.7555/JBR.34.20200700

154. Rasheed K, Qayyum A, Qadir J, Sivathamboo S, Kwan P, Kuhlmann L, et al. Machine learning for predicting epileptic seizures using EEG signals: a review. IEEE Rev Biomed Eng. (2021) 14:139-55. doi: 10.1109/RBME.2020.3008792

155. Pinto MF, Leal A, Lopes F, Dourado A, Martins P, Teixeira CA. A personalized and evolutionary algorithm for interpretable EEG epilepsy seizure prediction. Sci Rep. (2021) 11:3415. doi: 10.1038/s41598-02182828-7

156. Geng D, Alkhachroum A, Melo Bicchi M, Jagid J, Cajigas I, Chen ZS. Deep learning for robust detection of interictal epileptiform discharges. J Neural Eng. (2021). doi: 10.1088/1741-2552/abf28e

157. Maier A, Syben C, Lasser T, Riess C. A gentle introduction to deep learning in medical image processing. Zeitschrift Med Phys. (2019) 29:86101. doi: 10.1016/j.zemedi.2018.12.003

158. Karoly PJ, Ung H, Grayden DB, Kuhlmann L, Leyde K, Cook MJ, et al. The circadian profile of epilepsy improves seizure forecasting. Brain. (2017) 140:2169-82. doi: 10.1093/brain/awx173

159. Kiral-Kornek I, Roy S, Nurse E, Mashford B, Karoly P, Carroll $\mathrm{T}$, et al. Epileptic seizure prediction using big data and deep learning: toward a mobile system. EBioMedicine. (2018) 27:103-11. doi: 10.1016/j.ebiom.2017.11.032

160. Leguia MG, Andrzejak RG, Rummel C, Fan JM, Mirro EA, Tcheng TK, et al. Seizure cycles in focal epilepsy. JAMA Neurol. (2021) 78:45463. doi: 10.1001/jamaneurol.2020.5370

161. Wagenaar JB, Worrell GA, Ives Z, Dümpelmann M, Litt B, Schulze-Bonhage A. Collaborating and sharing data in epilepsy research. J Clin Neurophysiol. (2015) 32:235-9. doi: 10.1097/WNP.0000000000000159
162. Lehnertz K, Litt B. The First International Collaborative Workshop on seizure prediction: summary and data description. Clin Neurophysiol. (2005) 116:493-505. doi: 10.1016/j.clinph.2004. 08.020

163. Klatt J, Feldwisch-Drentrup H, Ihle M, Navarro V, Neufang M, Teixeira C, et al. The EPILEPSIAE database: an extensive electroencephalography database of epilepsy patients. Epilepsia. (2012) 53:1669-76. doi: 10.1111/j.1528-1167.2012.03564.x

164. DiLorenzo DJ, Leyde KW, Kaplan D. Neural state monitoring in the treatment of epilepsy: seizure prediction-conceptualization to first-in-man study. Brain Sci. (2019) 9:1-18. doi: 10.3390/brainsci9070156

165. Clinic UoPM. IEEG Portal (2014). Available from: https://www.ieeg.org (Retrieved February 24, 2021).

166. Lehne M, Sass J, Essenwanger A, Schepers J, Thun S. Why digital medicine depends on interoperability. NPJ Dig Med. (2019) 2:79. doi: 10.1038/s41746-019-0158-1

167. Varmus H. Genomic empowerment: the importance of public databases. Nat Genet. (2003) 35(Suppl. 1):3. doi: 10.1038/ng1186

Conflict of Interest: TL is part of patent applications to detect and predict clinical outcomes, and to detect, manage, diagnose, and treat neurological conditions, epilepsy, and seizures. TL is co-inventor of the TriVox Health technology and Boston Children's Hospital might receive financial benefits from this technology in the form of compensation in the future. WB is named along with TL on a patent for epileptogenicity that is owned by Boston Children's Hospital.

The remaining author declares that the research was conducted in the absence of any commercial or financial relationships that could be construed as a potential conflict of interest.

Copyright (c) 2021 Bosl, Leviton and Loddenkemper. This is an open-access article distributed under the terms of the Creative Commons Attribution License (CC BY). The use, distribution or reproduction in other forums is permitted, provided the original author(s) and the copyright owner(s) are credited and that the original publication in this journal is cited, in accordance with accepted academic practice. No use, distribution or reproduction is permitted which does not comply with these terms. 